P.

\title{
EVALUACIÓN DEL TRABAJO COTIDIANO: UNA COMPETENCIA DEL DOCENTE EN EL AULA
}

\author{
Magíster Ronald Soto Calderón
}

\section{Resumen}

Este artículo pretende compartir aspectos generales sobre la evaluación del trabajo cotidiano en el aula, en él se expresan algunas ideas con respecto a la labor que debe desarrollar el docente en el aula, y la forma en que debe realizarlo. A su vez, se recomiendan algunas técnicas que permitan el mejor registro de la actividad del estudiante, de manera que la valoración que realice el docente responda a las características y desempeño del estudiante en el salón de clase, en relación con el logro de los objetivos propuestos y el proceso de enseñanza y aprendizaje que se desarrolla en el aula. Es importante recalcar la idea de que se resalta la importancia de la observación, como una técnica que favorece la realización de la tarea que le corresponde al docente de aula o de materia específica.

Palabras clave: TRABAJO EN EL AULA/ DOCENTES/ ESTUDIANTES/ PROCESO ENSEÑANZA APRENDIZAJE/

\section{Introducción}

Dentro de las funciones del docente está la de valorar el trabajo cotidiano del estudiante.

Esta tarea se suma a la serie de aspectos de los que debe encargarse el docente con la finalidad de llevar a cabo su labor diaria en el aula y el trabajo que en ella deben realizar los estudiantes a su cargo.

El trabajo cotidiano debe ser la respuesta del estudiante al resultado de la propuesta metodológica que ofrece el docente para el desarrollo de sus necesidades, inquietudes e intereses.

La evaluación del trabajo cotidiano debe motivar al estudiante y al docente a involucrarse en las actividades que se proponen y emergen de la situación de enseñanza y aprendizaje, y a la interacción que de ella se genera junto con los demás miembros que componen el acto educativo que se desarrolla en el aula.

\footnotetext{
${ }^{1}$ Magister en Educación Especial -ICSE- Universidad Pontificia de Comillas, Madrid, España; Magister en Evaluación Educativa de la Universidad de Costa Rica; Sub director y profesor de la Escuela de Orientación y Educación Especial de la Universidad de Costa Rica; Profesor de la División de Educación Básica del Centro de Investigación y Docencia en Educación de la Universidad Nacional. Profesor de la Maestría en Estudios Interdisciplinarios sobre Discapacidad con mención en Sordoceguera y Discapacidad Múltiple. e-mail: ronaldsc@cariari.ucr.ac.cr
} 
Más allá de un momento que se observa y se valora en la clase durante el día lectivo, la evaluación del trabajo cotidiano debe tener un sentido de encuentro y análisis de la realidad educativa que se vive en el aula, debe permitir evaluar la práctica pedagógica que lleva a cabo el docente, y la forma en que ésta permite encausar los procesos educativos hacia una experiencia enriquecedora y pertinente.

El docente y el estudiante deben ser co-participantes de la experiencia de aula, y por lo tanto, generadores de experiencias que permitan el mejor desarrollo del hecho educativo, el cual motive la dinámica en el funcionamiento del aula.

Por los aspectos antes mencionados, se considera importante que el docente comprenda su papel en el proceso de evaluación del trabajo cotidiano y que esto lo motive a ser cada vez más observador de su experiencia diaria, lo que le permitirá desarrollar mejores estrategias de trabajo e interacción en el aula.

\section{La observación como parte del trabajo cotidiano}

La labor del docente en el aula va más allá de aplicar técnicas y desarrollar metodologías de enseñanza. Es más que eso, implica conocer las interacciones de sus estudiantes en un espacio donde se comparten múltiples subjetividades, tanto las que aporta el docente como elemento guía del proceso, como aquellas que aporta cada estudiante, (ambos actores del proceso de enseñanza y aprendizaje).

De esta manera, el docente debe ser un observador asiduo, que lea aquello que no escriben ni expresan verbalmente sus estudiantes, sino que lo representan mediante su acción en el espacio de aula que se comparte. Así, el docente, más que un facilitador del aprendizaje, se convierte en un asesor o supervisor que propone experiencias y comparte momentos que favorecen tanto el desarrollo cognitivo de la persona, como su interacción y socialización. De esta manera, el estudiante se verá identificado con procesos de concienciación que le permiten un mejor desarrollo de su potencial social y de la convivencia con otras personas, no únicamente de su edad, sino de los diferentes grupos que componen su comunidad.

Postic y De Ketele (1988) citan que

...el acto de observación es un proceso intelectual. Para mostrar la importancia cada 
vez mayor que tiene la inteligencia en la percepción, y por lo mismo en la observación, Piaget (1967) subraya el siguiente hecho: al comparar el peso de dos sobres de formatos diferentes, tenemos la tendencia a subestimar el más pequeño (p. 19).

La técnica de la observación, debe ser bien aprendida y desarrollada por los docentes, de tal manera que al implementarla en el aula, no se convierta en una técnica más, sino en una actividad que favorezca el desarrollo personal y profesional, y de esta manera, brinde el máximo apoyo a las personas con las que lleva a cabo su labor docente. Postic y De Ketele (1988) comentan que

... el aprendizaje de la observación no es solamente la progresiva apropiación de técnicas, está ante todo subordinado a una toma de conciencia de sí mismo como observador, de sus relaciones con la situación observada y de su implicación personal en la recogida de información (p. 205).

Muchos autores han escrito sobre la técnica de observación, y todos ellos han considerado que es una de las herramientas que mayor cantidad de información brinda a los docentes $u$ otros profesionales que la utilizan en su desempeño profesional. Lo anterior siempre y cuando se considere que cada experiencia de observación es diferente, y por lo tanto, debe ser planeada, estructurada y organizada de manera que permita obtener la información requerida, para tomar decisiones en función de los propósitos de la misma, en relación con un programa o proceso específico (Latorre y González ,1987; Postic y De Ketele,1988, Monereo, y Solé, 1999; Giné, 1999).

En este sentido Postic y De Ketele (1988, p. 25) citan que

... la evaluación continua, a lo largo del aprendizaje (ya sea la situación natural y ocasional) supondrá, pues, que la evaluación polariza su atención sobre los indicios conductuales que son signo claro de que los objetivos perseguidos se van alcanzando. Para ello, resulta indispensable un conocimiento previo de esos indicios comportamentales. Por lo demás, el educador, mediante su comportamiento verbal o no verbal, puede orientar la situación de tal forma que se convierta con naturalidad en revelador de las conductas esperadas de los alumnos.

Cada sesión de observación puede ser enriquecida por las experiencias en las que 
participa el profesional en educación; cada nueva situación que enfrenta el docente debe permitirle un análisis de las situaciones que se llevan a cabo en el aula y que de alguna manera requieren ser modificadas, cambiadas o sustituidas, lo cual favorece la realización del mejor esfuerzo para el logro de los objetivos propuestos en el plan curricular.

Por las razones antes mencionadas, es necesario que los docentes diseñen diferentes acciones en el aula que les permitan facilitar el registro de las situaciones o interacciones que se desarrollan en ella, con las cuales de alguna manera, se favorece el progreso individual o grupal de los alumnos y permite el desempeño efectivo del docente, en procura de ver satisfechas las necesidades tanto como de los estudiantes.

Las formas de recolección de la información le deben permitir a los docentes registrar las actuaciones de los estudiantes tal cual son, lo mismo que la manera en que ellos enfrentan su experiencia educativa; de la etapa del desarrollo por la cual atraviesan, y dando especial importancia a la atención y la comprensión de las características y particularidades de los estudiantes. De esta manera, se les brinda a ellos la oportunidad de desarrollar su máximo potencial en la vida o experiencia educativa, la cual se lleva a cabo en la escuela o el colegio, y en la que se espera tener éxito, siempre dentro de sus potencialidades, cualidades y necesidades (Latorre y González, 1987; Postic y De Ketele,1988, Monereo, y Solé, 1999; Giné, 1999).

La observación debe convertirse en una actividad diaria del docente, sin implicar un máximo de utilización de tiempo; por lo tanto, este puede diseñar diferentes instrumentos o medios, que le permitan el registro de información, y así facilitar de alguna manera la sistematización de la misma, lo cual haría del proceso un acto sencillo, dinámico y claro que posibilitaría el conocimiento de una realidad que se desarrolla en el aula y da indicios de dificultades o potencialidades que pueden ser utilizadas por el docente, para implementar experiencias enriquecedoras para todas las personas, favoreciendo de esta manera los procesos de realimentación entre los estudiantes y los estudiantes y el docente.

La observación por lo tanto, permitirá no solo una valoración del trabajo cotidiano, sino más bien, la identificación de las causas que provocan el comportamiento de los estudiantes en el aula, sea este adecuado o inadecuado, tanto al momento como a la acción que se está 
llevando a cabo mientras se realiza la observación. Así, el docente podrá favorecer estrategias que, bien implementadas, le permitirán lograr cambios en los estudiantes, no sólo en el ámbito cognitivo sino también en el aspecto socioemocional (Latorre y González, 1987; Postic y De Ketele,1988, Monereo, y Solé, 1999; Giné, 1999).

Por otro lado, la determinación del nivel de trabajo independiente de los estudiantes y el nivel de tolerancia para mantener la atención en la realización de una tarea, podrá determinarse mediante el uso de la observación, y así la respuesta del docente a las necesidades específicas y de aprendizaje de los estudiantes se verán satisfechas. Otro aspecto importante que puede observarse es el nivel de ayuda que requieren los estudiantes y cómo utilizar los recursos del aula para brindarles el apoyo, además de conocer la capacidad de trabajo en grupo de los estudiantes.

Ya se dijo que observar no es simplemente ver, sino que implica que se preste suficiente atención a las cosas y acciones importantes que se realizan en nuestro caso, para el proceso de enseñanza y aprendizaje. Para realizar esta acción de la manera más adecuada, es importante hacer uso de todos los sentidos, captando así los olores, los sonidos, los sabores, los tamaños y en general, observar el proceso de desarrollo del ser humano y sus implicaciones en el aprendizaje. En este sentido, hay que tener especial cuidado en no hacer interpretaciones de lo que se ve, sino más bien, convertir la tarea de la observación en un acto donde se describen las cosas tal y como se observan. Por esta razón se recomienda la construcción y utilización de instrumentos objetivos que favorezcan la obtención de información, para tomar decisiones con respecto al proceso de enseñanza y aprendizaje, y determinar el logro que ha tenido el estudiante en dicho proceso (Postic y De Ketele,1988; Giné, 1999).

Para poder llevar a cabo la observación, el docente debe diseñar registros que faciliten la recopilación y el procesamiento de la información, de tal manera que distinga entre lo relevante y lo irrelevante así, se podrá formar un cuadro claro y comprensible de la realidad observada y permitirá la toma de decisiones y apoyar las conclusiones a las que conlleva el proceso.

El docente puede utilizar para obtener y registrar información algunos instrumentos, entre 
los que se pueden mencionar:

\begin{tabular}{|c|c|}
\hline Información indirecta & Información directa \\
\hline Registro de observaciones & Anecdotarios \\
\hline Análisis de Tareas & Cuestionarios \\
\hline Escalas de producción escolar & Entrevista \\
\hline
\end{tabular}

Es importante aclarar que para efectos del trabajo cotidiano, el docente no debe registrar todo lo que ocurre durante la lección, sino aquellos momentos que le permitan de alguna manera evaluar formativamente el proceso de enseñanza que se lleva a cabo en el aula; de esta manera, puede contar con el diseño de una estrategia de evaluación que le permita realizar esta acción sin interrumpir el proceso de enseñanza; la evaluación del trabajo cotidiano debe estar coordinada y dirigida por el docente, quien al realizar una actividad o proceso específico tiene como fin determinar el dominio que ha adquirido el estudiante de " $X$ " tema, después de haber llevado a cabo un proceso de enseñanza; y donde el docente ha podido aplicar una metodología e implementado una acción mediatizadora o de facilitación; la evaluación del trabajo cotidiano está contenida como un objetivo del proceso, de manera que se logre implementar mediante una acción del docente y sus estudiantes.

\section{Aspectos importantes en la evaluación del trabajo cotidiano}

1. Claridad en cuanto al contenido o proceso que se pretende adquiera el estudiante.

2. Objetivo de la estrategia, método, o experiencia que se lleva a cabo en el aula.

3. Aspectos que darán evidencia de que el estudiante ha adquirido un contenido o un proceso.

4. El objetivo que justifica la evaluación del trabajo cotidiano.

5. Implementación de una estrategia para evaluar el trabajo cotidiano.

6. Elaboración de instrumentos que tomen en cuenta el contenido, objetivo, estrategia, método o experiencia y el objetivo de evaluación, al tiempo que favorezcan la recogida de información y su análisis.

\section{4.- Observando la dinámica del trabajo cotidiano del estudiante}

Para efectos de utilizar la observación en la evaluación del trabajo cotidiano, el docente debe tener en cuenta los siguientes aspectos 
1. Propósito de la observación a realizar.

En relación con este punto, el docente debe tener claro por qué se va a realizar la observación y el tipo de información que quiere obtener, de forma que en sus registro solo incluya los aspectos necesarios para apoyar su trabajo.

2. Objetivo de la observación.

Este debe ser claro y explícito en cuanto a lo que se quiere lograr con la observación. Muchas veces las personas al realizar esta actividad, registra información que resulta poco importante para la situación o trabajo que se observa.

3. Aspectos que serán observados.

El docente puede escribir un listado de aquellas conductas o trabajos del estudiante que quiere observar, de manera que si aparecen otras situaciones o conductas de la persona, le preste la atención que le merecen y las proponga en caso de ser necesario, para una posterior observación.

4. Estudiantes que serán observados.

Identificar a los estudiantes que serán observados es otro punto importante, ya que muchas veces los docentes se dedican a observar a otros miembros del grupo y no le brindan atención a aquellos para la que había sido propuesta la observación.

5. Determinar el foco de la observación.

Esta es una de las actividades más complicadas ya que el docente tiene que ubicar el lugar, espacio y conductas que debe realizar el estudiante, para obtener únicamente aquella información que le facilite al docente, la emisión de un criterio determinado.

6. Período que abarcará la observación.

El período dependerá de la duración de las tareas que se observan, y del nivel de trabajo de cada estudiante, en algunos casos puede ser un largo período, pero en otros será necesario un menor tiempo, e igualmente se conseguirá la información necesaria para valorar el trabajo cotidiano. 
7. Definir si se observa a todos los estudiantes o solo a parte de ellos.

Esto depende de la habilidad que tenga la persona para observar y del tipo y calidad de los registros que ella utilice, de forma que la labor se adecue a las características, necesidades e intereses del grupo y del docente.

8. Determinar las técnicas, e instrumentos que faciliten la recogida de información según los puntos $1,2,3,4,5,6$, y 7 . Estos pueden estar implementados o construidos, sino el docente podrá elaborarlos según sus criterios, objetivos y necesidades.

9. Determinar para efectos de la evaluación del trabajo cotidiano, la información que será tomada en cuenta para que pueda ser categorizada y analizada.

\section{Registros a utilizar por los docentes en la evaluación del trabajo cotidiano}

Hay modelos de los diferentes registros que aquí se recomiendan, y al final del artículo se ofrece bibliografía que podría enriquecer el quehacer del docente en este sentido (Latorre y González, 1987).

Entre los registros o técnicas que el docente puede utilizar se encuentran:

a.- Los anecdotarios, donde se incluyen el nombre del alumno, fecha, y las actividades 0 tareas que se observan o evalúan como parte del trabajo cotidiano.

b.- Listas de control. En una columna se escriben los objetivos que deben alcanzarse en un período a largo, mediano o corto plazo, y los indicadores que se desean evaluar en otra columna, durante un período determinado.

c.- Listas de cotejo. Se detallan en una columna todas aquellas actividades o pasos que completan una tarea o actividad, y en otra columna se escribe si el estudiante lo logra, si no lo logra o si está en proceso, así el docente puede valorar el grado de avance de los estudiantes.

Varios aspectos se deben tener en cuenta para su elaboración, entre ellos se pueden 
mencionar: haber seleccionado un objetivo del planeamiento didáctico, establecer los indicadores que serán observados, proponer una forma de utilizar la lista de cotejo, dar un sentido lógico a la colocación de los pasos, determinar e indicar cómo se desea medir los pasos.

Por ejemplo:

\begin{tabular}{|c|c|c|}
\hline \multirow[b]{2}{*}{ Indicadores } & \multicolumn{2}{|c|}{ Criterios } \\
\hline & Presente & Ausente \\
\hline 1. & & \\
\hline 2. & & \\
\hline 3. & & \\
\hline Total & & \\
\hline
\end{tabular}

d.- Escalas de valoración. En este caso los objetivos o indicadores que pretenden evaluarse se valoran en diferentes grados o niveles. Entre las escalas que se pueden construir se pueden mencionar las escalas gráficas, las escalas numéricas y las escalas descriptivas, pero se deben tener en cuenta las siguientes consideraciones:

i.- un ejemplar de escala para cada uno de los alumnos a los que se evalúa,

ii.- los objetivos que impliquen la adquisición de contenidos conceptuales, procedimentales y actitudinales,

iii.-en cada enunciado se describirá solo un aspecto para evaluar,

iv.- $\quad$ separar los enunciados en positivos y negativos, agruparlos por bloques o categorías, y

v.- establecer un número par de tramos, para valorar el mayor o menor progreso.

e.- Cuestionario. Puede ser otro instrumento para la recogida de información o de control de procesos y resultados de aprendizaje. Los cuestionarios se pueden realizar con preguntas cerradas, abiertas o mixtas.

f.- Sociogramas. Son gráficas que se obtienen de aplicar técnicas sociométricas. Mediante el sociograma se puede detectar la aceptación que un grupo tiene del alumno, o que el alumno posee de su grupo y el grado de cohesión del mismo, la existencia o inexistencia de un líder y la localización de estudiantes aislados y rechazados; en fin, es una 
representación gráfica que muestra la dinámica del grupo en una situación específica. En este sentido, para cada situación que quiere valorarse, se debe realizar un sociograma.

g.- Diario. Es de carácter más personal, es menos formal, pero es más rico en interpretaciones y reflejo de situaciones que suceden en el quehacer docente desde la subjetividad de cada profesor. Mediante este registro se pueden sistematizar experiencias, observaciones, y reflexiones de un participante y no existe ningún tipo de restricción en el diseño y elaboración del mismo.

h.- Grabaciones y filmaciones. En este caso el docente registra mediante el cassette 0 video. Esto le permite al docente no solo comparar sus registros con las grabaciones, sino también valorar de una mejor manera el desempeño de cada estudiante. Muchas veces el video permite el análisis de un aspecto o una situación que el educador ha omitido en el proceso de observación. No obstante, es necesario tomar todas las precauciones sobre la información que debe tener la persona, entre ellas, que está siendo grabada o filmada, y para qué es la grabación.

Estos son algunos ejemplos de los recursos que puede utilizar el docente en el proceso de evaluación del trabajo cotidiano, cada docente puede utilizar el formato que mejor le permita lograr el objetivo que se ha planteado en su trabajo.

\section{Analizando la información recolectada}

Al planificar la evaluación del trabajo cotidiano en forma adecuada, el docente puede además contar con información enriquecedora de la situación e interacción de los estudiantes en el ámbito grupal, transcribiendo y analizando todos aquellos datos recogidos que se generaron de la observación realizada durante la puesta en práctica de la experiencia de aprendizaje.

Es importante recordar que para fines de evaluación del trabajo cotidiano, el docente debe contar con guías de observación, listas de cotejo, escalas y otros, como técnicas de registro que le permitan una mayor sistematización de la información que resulta de la puesta en práctica de la experiencia realizada y faciliten posteriormente su análisis, pues esto le dará un mayor grado de credibilidad y fiabilidad al proceso; por lo tanto, este aspecto de la 
evaluación no puede ser una acción libre, asistemática e improvisada por parte del docente, sino más bien una actividad rigurosa, que enriquezca el criterio emitido por el maestro, y clarifique la toma de decisiones.

Se recomienda por esta razón, el uso de diferentes instrumentos diseñados por los docentes, que les permitan a éstos registrar el comportamiento o desempeño del estudiante, tomando en cuenta aspectos que deben ser valorados en los diferentes rubros. Esto permitirá la estructuración de los indicadores que facilitarán la valoración de las diferentes tareas o acciones que componen el trabajo cotidiano.

En este sentido, el docente debe tener muy claro qué aspectos del trabajo cotidiano va a evaluar. Esta evaluación puede ser desde los aspectos en los cuales se involucran los valores, hasta el desarrollo de actividades individuales y grupales relacionadas con el logro de objetivos de los planes de estudio propuestos por el Ministerio de Educación. Estos procesos le permitirán al docente identificar adecuadamente los rubros que evalúa, y un análisis cualitativo posterior de la información, de manera que la toma de decisiones con respecto al trabajo cotidiano del estudiante pueda tener un carácter más objetivo; situación que le permitirá al estudiante cambiar su participación en las actividades y procesos que se desarrollan en el aula.

El docente además, debe estar en capacidad de identificar diferentes situaciones que alteran o cambian el trabajo cotidiano de los estudiantes, quienes en algún momento, podrían estar pasando por situaciones que afectan su desempeño, participación e incorporación al trabajo de aula. Algunas veces, los estudiantes dan indicativos de problemas emocionales, afectivos y de salud; el docente debe estar preparado para valorar las implicaciones que estos problemas representan en la actuación de sus alumnos.

Como se puede ver, este proceso de evaluación corresponde especialmente a una evaluación formativa, la cual de alguna manera, si se le da seguimiento, podría aportar la información que se requiere para tener un perfil pertinente de los estudiantes y su desempeño en el aula, no solo a nivel cognitivo sino también en sus interacciones grupales, tanto con respecto a los compañeros como a los docentes de las diferentes materias que se imparten en la educación regular de primaria. 
Por otro lado, es importante tener en cuenta que para realizar la evaluación del trabajo cotidiano, se puede elaborar un instrumento general para las diferentes materias que cursa un estudiante en la escuela; sin embargo, si el trabajo cotidiano debe evaluarse en las diferentes materias, es necesario el uso de instrumentos específicos para cada una, o sea, un instrumento para matemática, otro para ciencias, otro para música y así sucesivamente, hasta abarcar todas las materias que componen los planes de estudio. De esta manera, se podrá ser más objetivo con respecto al desempeño del estudiante en cada materia; sin embargo, los instrumentos que se diseñen deben permitir analizar tanto datos cuantitativos como cualitativos, de manera que no prime solamente un tipo de valoración.

En relación con lo anterior, se debe tener en cuenta que la información recolectada solo adquiere relevancia en tanto se tiene conocimiento de lo observado, quién fue observado y la forma en que se obtuvo la información. Esto requiere que los registros se realicen simultáneamente al momento en que se observa o entrevista al estudiante que está siendo evaluado en su trabajo cotidiano. En este sentido, cuando el docente está impartiendo lecciones, puede asignar trabajo independiente y observar lo que ocurre en el aula o con el estudiante, puede también utilizar claves de registro de la información, por ejemplo, marcas en la pizarra o un cuaderno o registro, o en algún documento que haya implementado para el registro de información, en fin, existen muchos recursos que podrían facilitar el registro de la información; y todo dependerá de la organización e implementación de las actividades en el aula y de lo que el docente vaya a observar.

\section{A manera de conclusión}

La evaluación del trabajo cotidiano debe ser competencia del docente de aula o materia, pues le permite determinar el proceso de avance y logro que tiene cada estudiante con respecto a la materia que se desarrolló dentro del plan de estudios que propone el Ministerio de Educación en los diferentes niveles. Sin embargo, esta evaluación no debe convertirse en una tarea ardua del docente, sino que debe verse dinamizada por las acciones o instrumentos que utilice para llevar a cabo esta función del quehacer del profesional en educación.

El docente no debe abordar esta actividad evaluativa como una simple acción que complete una calificación que debe ser asignada a un estudiante, sino más bien, como una 
acción que le permita identificar los procesos que realiza en el aula y el grado de logro de los objetivos planteados en el programa; también le puede ayudar a valorar las acciones metodológicas que ha utilizado en el desarrollo de las diferentes temáticas en la clase.

El trabajo cotidiano por lo tanto, se convierte en una labor que favorece la obtención de información no solo con respecto al desempeño del estudiante en el aula, sino también información que le permita al maestro realimentar su práctica docente, aspectos que beneficiarán las futuras experiencias en las aulas.

Por otro lado, el docente estará en capacidad de poner en práctica el diseño de instrumentos que posteriormente serán enriquecidos en función de las observaciones de los estudiantes, la experiencia de la aplicación de los mismos por parte del docente y los procesos de análisis que dé la información obtenida con los instrumentos aplicados.

Como se puede ver esta labor enriquecerá más la experiencia docente y le permitirá una actualización de la misma, al tiempo que hará de la evaluación del trabajo cotidiano una experiencia rica y provechosa.

\section{Referencias}

Giné, C. (1999) "La evaluación psicopedagógica”. En Marchesi, A. Coll, C. y Palacios, J. Desarrollo psicológico y educación. III Trastornos del Desarrollo y necesidades educativas especiales. 2ª . Edición. España: Alianza.

Guimaraes, C. S. y Vargas, C. R. (1996) Conociendo la comunidad. Cartago, Costa Rica: A.G. COVAO.

Latorre. A. y González, R. (1987) El maestro investigador. Barcelona, España: Editorial Tao.

Monereo, C. y Solé, I. (1999) El asesoramiento psicopedagógico: una perspectiva profesional y constructivista. España: Editorial Alianza.

Postic, M. Y De Ketele, J. (1988) Observar las situaciones educativas. Madrid: Narcea, S.A. de Ediciones. 